\title{
Impact des installations côtières sur l'évolution du littoral de Mohammedia (Maroc)
}

VIII ${ }^{\text {èmes }}$ Journées Nationales Génie Civil - Génie Côtier, Compiègne, 7-9 septembre 2004

\author{
Mohammed Idrissi ${ }^{(a)}$ Ahmed Hourimeche ${ }^{(b)}$, \\ Vincent Rey ${ }^{(\mathrm{c}),}$ Mohammed Chagdali ${ }^{(\mathrm{d})}$
}

\author{
(a)Doctorant, Université Hassan II - Mohammedia et Université du Sud Toulon-Var \\ (b)Professeur Habilité, Université Hassan II - Mohammedia \\ (c)Maître de Conférences, Université du Sud Toulon-Var \\ (d)Professeur, Université Hassan II - Mohammedia
}

\section{Résumé :}

L'augmentation des activités industrielles et l'urbanisation croissante ont conduit ces dernières années à la construction de nombreux ouvrages le long du littoral de Mohammedia, conduisant à des modifications dans la distribution des sédiments et à une dégradation de l'environnement naturel de certaines plages. La comparaison entre les positions du trait de côte, observées à partir de photographies aériennes, en 1969, 1986 et 1997 montrent une forte évolution des plages, de 0,6 à 1,5 mètres par an (en accrétion ou érosion), et pouvant atteindre 3 mètres par endroits, notamment près des ports et des jetées.

Une étude des variations saisonnières du profil de la plage du bas de l'estran à la dune bordière du littoral de Mohammedia, menée durant la période 2001-2002, a montré un engraissement significatif au niveau du port et de la plage de Mohammedia, et une érosion entre Manesmann et l'Oued Nfifikh. Cependant, les résultats de calculs d'évolution à long terme basée sur l'hypothèse d'équilibre des profils de plage en terme de bilan sédimentaire se sont révélés être notablement inférieurs à ceux déduits de l'étude d'évolution annuelle, ils s'accordent cependant à démontrer les effets des structures implantées sur les récents changements de la dynamique dans cette zone.

\section{Abstract :}

Due to a significant industrial activity and an increasing urbanization, numerous coastal installations have been built along the shore, leading to modifications in the sediments distribution, and a degradation of the natural environment of some beaches. Comparison of the shorelines obverved for the years 1969, 1986 and 1997, using aerial photographs, showed a general tendency of shoreline evolution in the range $0,6-1,5 \mathrm{~m} /$ year (either accretion or erosion), it can exceed locally $3 \mathrm{~m} /$ year.

The study of the seasonal variations of the beach profiles of the Mohammedia littoral were carried out according to a well defined squaring during the period 2001-2002 showed a significant fattening for Mohammedia port and beach, and a thinning down between Manesmann and the Oued Nfifikh.

However, by use of an equilibrium beach profile hypothesis for the shoreline changes, and of significant wave data for the calculation of the closure depth, we observed that the estimate of the beach changes, in terms of sediment budgets, through the medium term (annual) study is significantly higher than the mean erosion observed during the last decades, but both agree to demonstrate the impact of the structureson the recent nearshore dynamics changes. 


\section{Introduction}

Le littoral de Mohammedia est une zone particulièrement intéressante pour l'étude et la compréhension de l'influence des processus naturels et anthropiques sur la dynamique sédimentaire. L'augmentation des activités industrielles et l'urbanisation croissante ont conduit ces dernières années à la construction de nombreux ouvrages le long de la côte, conduisant à des modifications dans la distribution des sédiments et à une dégradation de l'environnement naturel de certaines plages.

L'évolution spatio-temporelle de la plage, aussi bien d'un point de vue des répartitions granulométriques des sédiments que des profils de plage est présentée dans une première partie. Les données obtenues à long terme et à moyen terme sont ensuite analysées et les bilans sédimentaires calculés. Les données issues de l'évolution du trait de côte et celles issues des mesures sur une année sont comparées et les évolutions ainsi que les différences discutées.

\section{Données sédimentologiques et d'évolution des plages}

\subsection{Analyse des sédiments}

\subsubsection{Caractérisation des sables}

Pour la caractérisation des sables du littoral Mohammedia, nous avons utilisé les indices granulométriques de Folk et Ward ${ }^{1}$, exprimés en unité Phi. L'analyse granulométrique des sables prélevés le long de ce littoral pendant la période 2001-2002 montre un large spectre granulométrique allant des sédiments fins aux sédiments grossiers (Fig. 1). Les sables sont très bien classés et montrent généralement des asymétries positives indiquant une dominance de la fraction grossière. Ces résultats sont comparables à ceux obtenus par Khiri ${ }^{2}$ et Hourimeche $^{3}$. Les plages du secteur Ouest (port de Mohammedia, plage Mohammedia) sont constituées essentiellement de sédiments fins. Ce secteur Ouest, protégé par la jetée du port de Mohammedia, reçoit des vagues de faible énergie qui n’ont aucun effet érosif sur ces plages. Les plages du secteur est (plage Mannesmann, plage Monika, plage Sablette) sont formées de sables moyens à grossiers. Ce secteur Est, exposé à l'action des vagues du large, subit une forte érosion et un vannage des sables fins vers le large (Idrissi ${ }^{4}$, Idrissi et $\mathrm{l}^{5}$ ).

\subsubsection{Minéralogie des sables}

L'analyse des minéraux lourds montre un cortège minéralogique dominé par l'augite (58 à 76\%). L'hornblende arrive en deuxième position avec 15 à 27\%. Le zircon est présent avec une proportion non négligeable (4 à 9\%). Les autres minéraux lourds (pumpellyite, épidote, disthène, tourmaline et grenat) sont représentés avec des proportions allant de 1 à $5 \%$ (Fig. 1). L'augite provient essentiellement des basaltes triasiques affleurants au Sud-Est du secteur étudié, dans la vallée de l'oued Nfifikh (Hourimeche ${ }^{3}$ ). Certaines augites ainsi que les minéraux magmatique et métamorphique qui leurs sont associés proviendraient des régions Nord par la dérive littorale; à partir des sédiments déversés dans l'océan atlantique par les différents oueds qui drainent les massifs du moyen Atlas, du Maroc central et de la méséta côtière. 


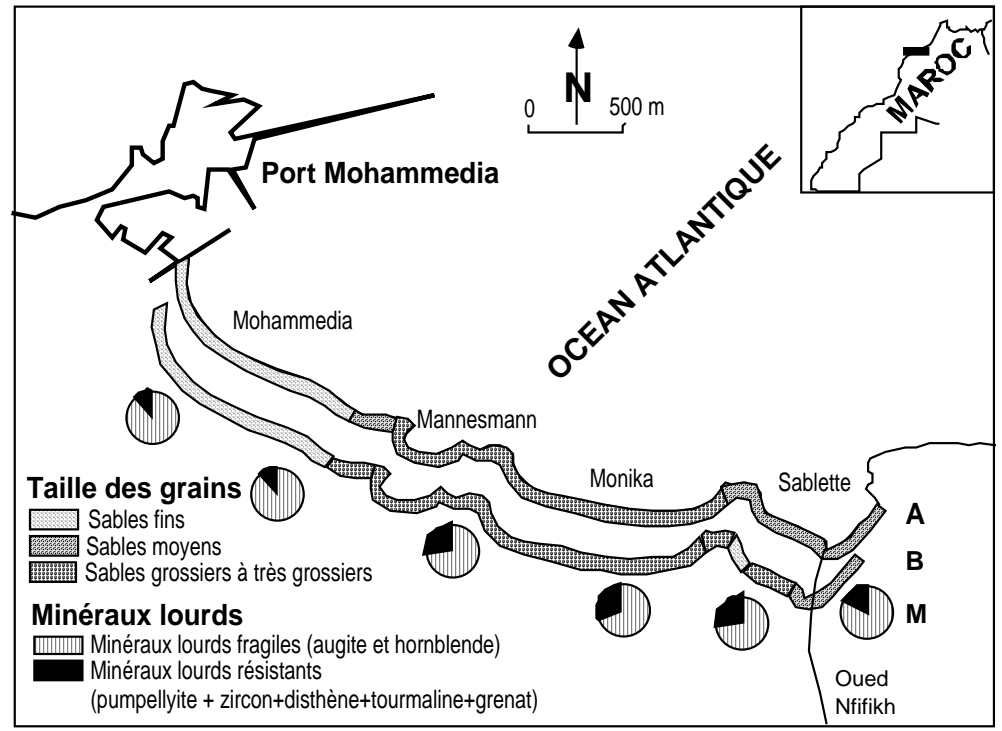

Figure 1: Répartition granulométrique et minéralogique des sables du littoral de Mohammedia $A$ : période hivernale $\quad B:$ période estivale $\quad M$ : minéraux lourds

Le groupement des minéraux lourds en minéraux résistants et minéraux fragiles montre une dominance des minéraux fragiles (augite, hornblende) au niveau des plages du secteur Ouest (port de Mohammedia, plage Mohammedia) qui correspondent à un secteur de faible agitation. L'augmentation des proportions des minéraux lourds résistants (pumpellyite, zircon, tourmaline, disthène, grenat) au détriment de l'augite, au niveau des plages du secteur Est (plage Mannesmann, plage Monika, plage Sablette), prouve bien que ces plages subissent une forte agitation.

\subsection{Evolution des plages}

\subsubsection{Evolution du trait de côte}

L'analyse des photographies aériennes du littoral de Mohammedia, des années 1969, 1986 et 1997, a permis de suivre l'évolution des plages de toute la zone étudiée. Cette visualisation graphique des photographies aériennes a permis de subdiviser la zone d'étude en deux secteurs (Fig.2).

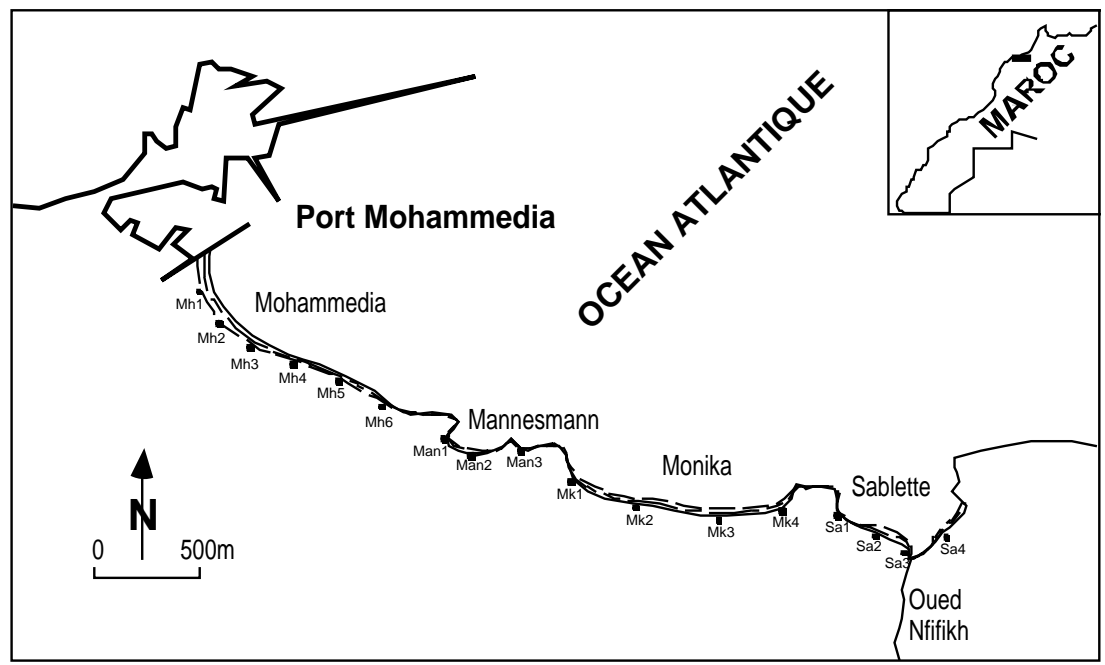

Figure 2 : Evolution du trait de côte du littoral de Mohammedia (Maroc) entre 1969 et 1997 
- Un secteur Est en érosion: les plages de ce secteur Est (plage Monika, plage Mannesmann) subissent un recul continu du trait de côte qui se manifeste par la formation de croissants de plage, l'érosion de la dune bordière, l'apparition de platier rocheux au milieu de l'estran, et de cordons de galets de disposition perpendiculaire à la côte. Ce recul est estimé de 42 à $84 \mathrm{~m}$ pendant 28 ans, soit un taux de démaigrissement compris entre 1,5 et $3 \mathrm{~m} / \mathrm{an}$. L'érosion de ce secteur Est est due à son exposition à l'action de la houle qui se manifeste par une forte amplification et convergence des vagues, en relation avec la présence des dorsales sous marines et des hauts fonds au large de la plage Monika (Aït Laamel ${ }^{6}$ ). Le même phénomène d'érosion côtière a été constaté dans la baie de Tanger (Migniot ${ }^{7}$ ), dans la baie d'Agadir (Charrouf ${ }^{8}$ ). Cependant, la plage Sablette n'a subi qu'un léger recul de son trait de côte au cours de la même période d'étude. Ceci est en rapport avec sa situation au niveau de l'embouchure de l'oued Nfifikh qui apporte des sédiments fins à moyens et par sa morphologie en entonnoir qui joue un rôle important dans l'amortissement et la dissipation de l'énergie des vagues.

- Un secteur Ouest en progradation : les plages de ce secteur subissent un engraissement, matérialisé par un ensablement du port de Mohammedia et une progradation continue du trait de côte de la plage Mohammedia. Le taux d'engraissement est de plusieurs dizaines de mètres sur 28 ans. Ce secteur, protégé par la jetée du port de Mohammedia, montre des vagues qui ont un effet érosif plus faible. L'engraissement et la forme arquée des plages du secteur Ouest sont dus à la rotation de la houle autour de l'extrémité de cette jetée principale qui se traduit par la modification de sa direction et le transfert de son énergie vers la zone protégée. Ce phénomène de diffraction entraîne la création d'un courant qui ramène les sables depuis la plage Monika vers la plage Mohammedia et qui est renforcé par le courant de dérive littorale, circulant du Nord vers le Sud le long de frange côtière. De ce fait, le secteur Ouest du littoral Mohammedia, protégé par la jetée principale, se comporte comme une pompe aspirant en permanence les sables du secteur Est. Ce transit latéral des sédiments est responsable de l'édification au niveau de la plage Mannesmann de plusieurs corps sédimentaires (tombolos), reliant la côte au platier rocheux. L’élargissement et la surélévation de la plage Mohammedia sont en relation avec la présence de l'épi transversal qui protège le port de Mohammedia et qui oblige le courant de dérive littorale à déposer une grande partie de sa charge sédimentaire au niveau de ce secteur Ouest.

\subsubsection{Variation des profils de plage}

Le nivellement des estrans des plages du littoral de Mohammedia, réalisé selon un quadrillage bien défini au cours de l'année 2001/2002, a permis d'acquérir les évolutions cross-shore en 17 points distants d'environ 100 à $300 \mathrm{~m}$ entre le port de Mohammedia et l'oued Nfifikh. Deux exemples d'évolution de profil, le premier en accrétion, le second en érosion, sont présentés Fig. 3 et Fig. 4. Les 17 profils montrent des évolutions assez homogènes le long du profil, avec déplacement sans modification notable de l'allure du profil.

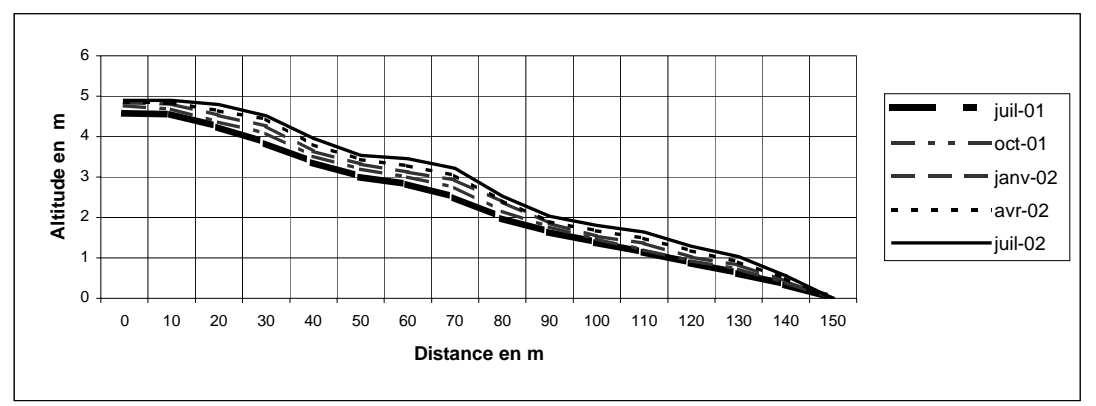

Figure 3 : Exemple de profils de plage en accrétion (point Mh2, plage Mohammedia) 


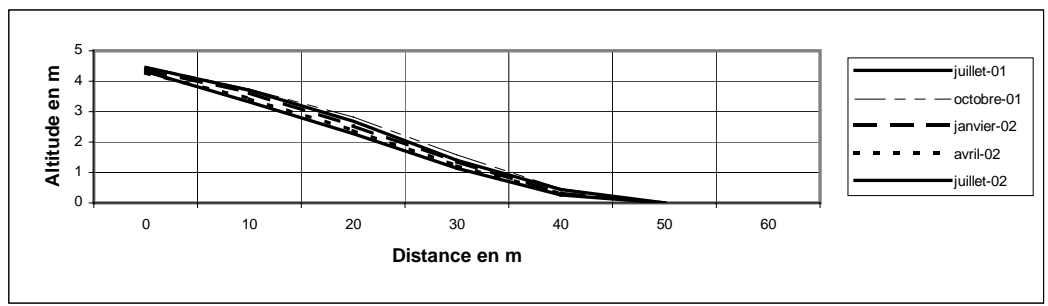

Figure 4 : Exemple de profils de plage en érosion (point Mk2, plage Monika)

Le principe de cette étude est basé sur un nivellement direct effectué trimestriellement afin d'avoir les variations d'altitudes sur les plages pendant la période d'étude. Les profils mesurés couvraient tout l'estran depuis des points connus et bien matérialisés sur la corniche jusqu'au bord de la mer à marée basse. Les mesures, espacées de $10 \mathrm{~m}$ pour chaque profil, étaient faites par nivellement direct avec un niveau Wild N2.

\section{Analyse des évolutions morphologiques}

\subsection{Variation des profils de plage}

L'étude menée en 2001-2002 a montré un appauvrissement en sables de toutes les plages du secteur Est de la zone d'étude. Les données des profils permettent de calculer des bilans sédimentaires. L'accrétion ou l'érosion des plages en terme de volumes est présenté dans le tableau 1. Au cours de cette période d'étude, c'est la plage Monika qui a subi le plus cet appauvrissement, avec $72977 \mathrm{~m}^{3}$. Par contre, cette étude topographique a montré un engraissement des plages du secteur Ouest pendant la période d'étude. La plage Mohammedia a enregistré un gain de sables de l'ordre de $41848 \mathrm{~m}^{3}$. Cependant, le port de Mohammedia qui subit un ensablement nécessite des travaux de dragage afin de maintenir une activité normale. Le volume total de sables perdus au niveau du littoral de Mohammedia, au cours de cette période d'étude, est évalué à $91269 \mathrm{~m}^{3}$.

Tableau 1: Estimation du volume total (en $\mathrm{m}^{3}$ ) de sables perdus du littoral Mohammedia

\begin{tabular}{|l|l|l|l|l|l|}
\hline$\left(\mathrm{V}_{\mathrm{ii}+1}\right)^{\mathrm{j}+1}$ en $\mathrm{m}^{3}$ & $\begin{array}{l}\text { Période } \\
(\mathrm{i}=1)\end{array}$ & $\begin{array}{l}\text { Période } \\
(\mathrm{i}=2)\end{array}$ & $\begin{array}{l}\text { Période } \\
(\mathrm{i}=3)\end{array}$ & Période (i=4) & $\begin{array}{l}\text { Total des } \\
\text { volumes }(\mathrm{V})^{\mathrm{j}+1}\end{array}$ \\
\hline Mohammedia & +11872 & +9438 & +10364 & +10173 & +41848 \\
\hline Mannesmann & -8462 & -10578 & -11436 & -10532 & -41009 \\
\hline Monika & -15428 & -21643 & -18248 & -17657 & -72977 \\
\hline Sablette & -4367 & -6734 & -2657 & -5372 & -19131 \\
\hline $\begin{array}{l}\text { Total des } \\
\text { volumes }\left(\mathrm{V}_{\mathrm{ii}+1}\right)\end{array}$ & -16385 & -29517 & -21977 & -23389 & -91269 \\
\hline
\end{tabular}




\subsection{Evolution du trait de côte}

L'hypothèse de Bruun ${ }^{9}$ permet de supposer que les plages en érosion ou en accrétion se déplacent parallèlement à elles-mêmes, sans déformation de leur profil à une échelle de l'ordre d'une année. Le volume sédimentaire érodé ou apporté se déduit donc de la position du trait de côte, à partir du moment où la hauteur h de mobilisation du sédiment est connue. Cette hauteur est la différence entre la hauteur maximale de la mer par surcote, et la profondeur de fermeture, valeur de la profondeur d'eau au delà de laquelle les mouvements sédimentaires n'ont plus d'influence sur la morphodynamique. Cette profondeur D peut être estimée à partir de la formule d' Hallermeier ${ }^{10}$ :

$D=\left[2.28-10.9 \frac{H_{0}}{L_{0}}\right] H_{0}$

où $\mathrm{H}_{0}$ et $\mathrm{L}_{0}$ sont la hauteur significative et la longueur d'onde des houles au large. En se basant sur les études de la SOGREAH ${ }^{11}$, les houles les plus significatives ont une période de $\mathrm{T}_{0}=15 \mathrm{~s}$, une hauteur $\mathrm{H}_{0}=3.4 \mathrm{~m}$, avec une probabilité d'occurrence d'environ $10 \%$. Sachant que le marnage est de $4 \mathrm{~m}$, et si l'on prend une surcote de $2 \mathrm{~m}$, on en déduit h. Les données à long terme nous ont ainsi permis de déterminer les variations annuelles moyennes des volumes sédimentaires le long de la plage (Fig. 5).

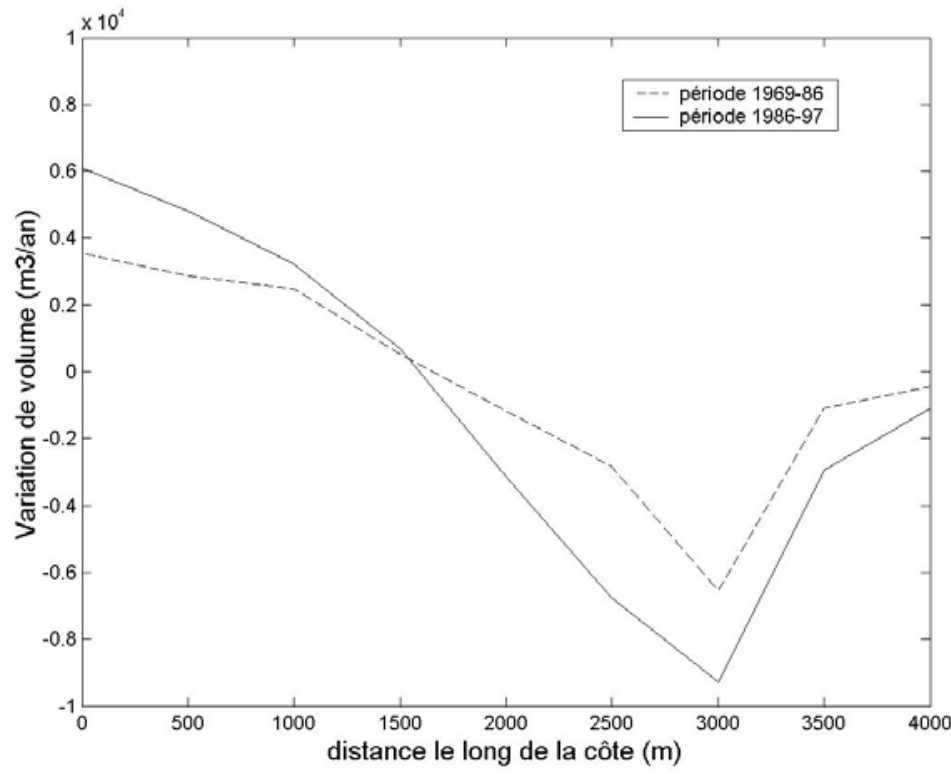

Figure 5: Variations annuelles moyennes des volumes sédimentaires

Pour les plages de Mohammedia et de Monika, ces variations annuelles sont estimées respectivement à $9414 \mathrm{~m}^{3}$ et $-10911 \mathrm{~m}^{3}$ pour la période $1969-86$ et $14793 \mathrm{~m}^{3}$ et $-20090 \mathrm{~m}^{3}$ pour la période 1986-97. Les erreurs dues au choix de la période et de la hauteur significative restent assez faibles ( $\pm 1 \%$ à $\mathrm{H}_{0}$ constant si $\mathrm{T}=\mathrm{T}_{0} \pm 3 \mathrm{~s}, \pm 25 \%$ à $\mathrm{T}_{0}$ constant si $\mathrm{H}=\mathrm{H}_{0} \pm 1.6 \mathrm{~m}$ ).

\subsection{Comparaison des données annuelles et long terme}

Les données recueillies en 2001-2002 (voir Tableau 1) prévoient donc des évolutions annuelles des plages de Mohammedia et de Monika (respectivement $41848 \mathrm{~m}^{3}$ et $-72977 \mathrm{~m}^{3}$ ) de l'ordre de trois fois supérieures à celles calculées à partir des données du trait de côte (respectivement $14793 \mathrm{~m}^{3}$ et $-20090 \mathrm{~m}^{3}$ par an en moyenne pour la période 1986-97). 
D'un point de vue qualitatif, une explication serait l'augmentation régulière de la morphodynamique liée aux implantations successives de structures le long de cette côte. En effet, l'érosion s'est accrue entre 1969 et 1997 d'après les calculs du paragraphe précédent, ce qui est compatible avec les résultats de la période 2001-2002 qui montrent une érosion annuelle plus importante que celle observée en moyenne sur la période 1986-1997. Une information de la position du trait de côte actuel permettrait de vérifier (ou d'infirmer) l'augmentation du processus ces dernières années.

D'un point de vue quantitatif, la différence (de l'ordre d'un facteur 3) semble cependant trop importante pour n'être expliquée que par des processus d'érosion (plage de Monika) et d'accrétion (plage de Mohammedia) croissants ces dernières années. Les calculs basés sur les données topographiques de la période 2001-2002 ne tiennent compte que des variations dans les zones de haut de plage et d'estran. Si on suppose que ces variations sont associées aux évolutions "cross-shore » liées aux épisodes de tempêtes, alors les variations de volume sédimentaire sur un profil de plage sont surestimées. Si on suppose que la plage est en équilibre (profil de Bruun), alors les variations de volume sont sous-estimées (à cause d'un décalage en bloc du profil (d'équilibre) de plage dans ses parties émergée et immergée). D’après les écarts entre les résultats obtenus soit par données topographiques soit par données du trait de côte, il apparait donc que les analyses volumétriques des données recueillies sur des échelles de temps de l'ordre d'une année ne sont pas représentatives de l'érosion pluriannuelle basée sur la position moyenne du trait de côte.

\section{Conclusion}

Les études sédimentologique et topographique, couplées à l'analyse des photographies aériennes du littoral de Mohammedia, ont permis de mettre en évidence deux secteurs morphosédimentaires. L'évolution morphodynamique de chacun de ces deux secteurs est en relation avec les facteurs naturels et les travaux d'aménagement réalisés au niveau de ce littoral.

Un secteur en démaigrissement localisé dans la partie Est du littoral de Mohammedia. Les plages de ce secteur (plage Mannesmann, plage Monika, plage Sablette) sont constituées essentiellement de sables moyens à grossiers avec des proportions non négligeables de minéraux lourds résistants. Ce secteur, exposé à l'action des houles, est caractérisé par une forte amplification et convergence des vagues et correspond à une zone de forte à très forte agitation (zone d'érosion).

Un secteur en engraissement situé dans la partie Ouest du littoral de Mohammedia. Les plages de ce secteur (plage Mohammedia, port de Mohammedia) sont constitués de sables fins avec une prédominance de minéraux lourds fragiles. Ce secteur Ouest, protégé de l'action des vagues par la jetée du port, reçoit des vagues réfractées et correspond à une zone de faible agitation (zone d'ombre). La présence de l'épi transversal à l'extrémité ouest de la plage Mohammedia a permis l'élargissement et la surélévation des plages du secteur Ouest. Cet effet s'est accru dans la période 1986-1997 suite à la construction de la grande jetée (longueur 2585 m) du port de Mohammedia.

Les données d'évolution annuelle et pluri-annuelles ont été comparées, en supposant qu'on pouvait définir un profil d'équilibre dynamique pour les calculs d'évolution à long terme, et que les changements de profils des plages au dessus du niveau de plus basses mers étaient représentatifs des mouvements sédimentaires annuels. Il s'est avéré que les résultats sont compatibles d'un point de vue qualitatif mais que la quantité de sable déplacé est d'un ordre trois fois supérieur si l'on se réfère aux données annuelles plutôt que pluri-annuelles. 
Des données de position du trait de côte actuel semblent être une donnée supplémentaire nécessaire pour expliquer si ce sont plus les modes de calculs que les constructions d'ouvrages récents qui sont à l’origine de ces différences.

\section{Références}

1.Folk, R.L., \& Ward, W.C., 1957. Brazos river bar: a staday in the significance of grain size parameters. J. Sedim. Petrol., 27(1), pp. 3-26.

2.Khiri F. (1995). Etude géomorphologique et sédimentologique du littoral de Mohammedia. Mém. CEA Sciences de la mer, Univ. Chouä̈b Doukkali, Fac. Sci. El Jadida ; $61 \mathrm{p}$.

3.Hourimeche A. (1999). Etude sédimentologique des formations littorales plioquaternaires et des dépôts côtiers actuels de la région d'Essaouira - comparaison avec la région de Casablanca -. Thèse Doctorat d’Etat. Fac. Sc. Ben M’Sik, Casablanca ; 271 p.

4.Idrissi M. (2000). Etude sédimentologique et évolution morphologique actuelle de la zone côtière Casablanca-Mohammedia (Maroc). Mém. DESA. OGEA. Univ. Mohammed VAgdal, Rabat ; 104p.

5.Idrissi M., Hourimeche A. et Chagdali M. (2002). Impact de la houle sur l'évolution morphologique et sédimentolgique actuelle de la zone Casablanca-Mohammedia (Maroc). $19^{\text {th }}$ Colloquium of African Geology, 19-22 mars, El Jadida, Morocco, p. 102.

6.Aït Laamel M. (1999). Modélisation numérique de la propagation de la houle du large vers la côte. Mémoire DESA, Université Hassan II - Mohammedia, Fac. Scie. Ben Msik, Casablanca, Maroc, 45 p.

7.Migniot C. (1981). La défense des côtes, érosion et sédimentation en mer, les causes et les moyens d'action. Ecole Nationale des Ponts et des Chaussées. Laboratoire Central d'Hydraulique de France.

8.Charrouf L. (1989). Problèmes d'ensablement des ports marocains sur la façade atlantique. Leur impact sédimentologique sur le littoral. Thèse Univ. Paris Sud, 278.

9.Bruun P. (1954). Coast erosion and the development of beach profiles., Technical memorendum, 44, Beach erosion board US Army Corps of Engineers, Waterways Experiment Station, Vicksburg, MS.

10.Hallermeier H. (1981). A profile zonation for seasonal sand beaches from wave climate, Coastal Eng., 4, pp 253-277.

11.SOGREAH. (1984). Plage de Mohammedia, Etude d'impact de la jetée sur le littoral, Dossier 350-872-R4, SOGREAH -MAROC, Janvier 1984 